\title{
Critical care nurses' moral distress in end-of-life decision making
}

\author{
Saira Weinzimmer ${ }^{1}$ Susan M. Miller ${ }^{2,3}$, Janice L. Zimmerman ${ }^{2,3}$, J ay Hooker ${ }^{3}$, Stacey I sidro ${ }^{4}$, \\ Courtenay R. Bruce ${ }^{3,5}$
}

1. Rice University; Houston, TX, USA. 2. Weill Cornell Medical College; New York, NY, USA. 3. Houston Methodist Hospital, Houston Methodist Hospital System Biomedical Ethics Program, Houston, TX, USA. 4. The University of Texas M.D. Anderson Cancer Center, Houston, TX, USA. 5. Center for Medical Ethics \& Health Policy, Baylor College of Medicine, Houston, TX, USA.

Correspondence: Courtenay Rose Bruce. Address: Center for Medical Ethics \& Health Policy, Baylor College of Medicine, Houston, TX, USA. Email: crbruce@bcm.edu

Received: January 29, 2014

DOI : $10.5430 /$ jnep.v4n6p6
Accepted: March 28, $2014 \quad$ Online Published: April 9, 2014

URL: http://dx.doi.org/10.5430/jnep.v4n6p6

\section{Abstract}

Moral distress is a phenomenon in which a healthcare professional perceives an ethically preferable or morally right course of action to take, but internal or external constraints make it nearly impossible to pursue that course. Although healthcare professionals' moral distress has been studied for over twenty years, we have not reached a point of full understanding and appreciation of this complex phenomenon. Attempts to define moral distress and refine our understanding of it have been frustrated by tendencies to interchange or mislabel very distinct—yet related—concepts. A comprehensive knowledge base of root causes and the interrelationships between individual and team or system factors is timely and critical.

We qualitatively interviewed 29 healthcare professionals of various backgrounds in two intensive care units at a tertiary, academic medical center with the goal of understanding interrelationships between team and individual factors of moral distress. Herein, we report our findings with regard to nurses' moral distress and suggest a list of practical strategies to mitigate this moral distress.

\section{Key words}

Moral distress, Bioethics, Ethics, Patient care

\section{I ntroduction}

With technological innovation and advancement, the practice of medicine is increasingly fraught with ethical challenges. Moral distress occurs when a healthcare professional perceives an ethical course of action, but is prevented from pursuing it either by internal (personal) or external (institutional) limitations ${ }^{[1,2]}$. Moral distress is distinct from other related phenomena, such as burnout, ethical dilemmas, compassion fatigue, and general psychological distress ${ }^{[3]}$. These concepts are often used interchangeably in the literature, adding to the difficulty of studying moral distress. Moral distress is unique in that it involves a violation of personal values and disrupts moral identity. It induces feelings of sadness, frustration, anger, guilt, low self-esteem, depression, and anxiety, and it often manifests in job dissatisfaction and detachment ${ }^{[4-7]}$. 
Research into the causes and facets of moral distress has greatly increased in recent years. First, the concept has now been expanded to include all healthcare professionals and disciplines and no longer remains nursing-centric ${ }^{[4,8-10]}$. Second, moral distress has been given new definitional characteristics, such as clarification between "moral distress"-the immediate distress a clinician feels upon his or her inability to take ethical action—and "moral residue," the lingering distress that can remain for years after the situation has elapsed ${ }^{[2]}$. Recently, Epstein and Hamric coined the term "Crescendo Effect" to refer to the cumulative building of moral distress over time that occurs with repeated exposure to morally distressing cases ${ }^{[2,10]}$. Third, there has been renewed interest in the implications of moral distress and the effects it has on healthcare quality and efficiency. For instance, research efforts have focused on healthcare professionals' physical and emotional detachment from patients and cases during bouts of moral distress ${ }^{[3,6,11-15]}$. Prevalence of moral distress has also been studied in the context of staff retention rates ${ }^{[11,12]}$.

Several root causes of moral distress have been identified in the literature. Some root causes are externally-focused. Common examples include: administration of medically inappropriate treatment and concerns about communication between healthcare professionals or between patients and healthcare professionals ${ }^{[2,5,7,14,16]}$. The ethical climate in which clinicians work--determined by the institutional systems in place to resolve ethical issues--can heavily influence moral distress, although the nature of its influence remains uncertain ${ }^{[6]}$. Internal considerations include feelings of powerlessness or perceptions of power differentials between healthcare professionals ${ }^{[2]}$.

Though the literature has taken a wide survey of moral distress over the past 20 years, there are several important gaps in knowledge that must be addressed by further information. There has been a lack of investigation into the interconnections between individuals, unit/team, and system factors, and their relationship to the root causes of moral distress. In addition, research has largely focused on nursing; comparisons between professions have been limited ${ }^{[3,6,17]}$.

The purpose of this paper is to explore the gap in understanding the interconnections between individual and team factors involving moral distress. We used a qualitative method of in-depth individual interviews which allows for an initial exploration of such a multifaceted and complex concept. To our knowledge, this is the first moral distress study that used a case report methodology wherein interviewees were asked to describe their experiences with one particular clinical case identified as being morally distressing. Below, we outline our findings and provide a brief analysis of our results.

\section{Methods}

\subsection{Subjects}

Following institutional review board (IRB) approval, three cases from two intensive care units (ICUs) that were thought to be potentially morally distressing were selected by co-investigators. Three project co-investigators are medical or nursing directors in these two units and thus had firsthand, experiential knowledge of cases thought to evoke moral distress. Participants from these two ICUs were recruited using purposive sampling of healthcare professionals identified as being "key" players in these cases. Our principal goal in purposive sampling was to identify those healthcare professionals who would have intimate knowledge of the cases.

In total, 29 health care professionals were recruited and oral consent for participation was obtained using an IRB-approved script. Their professional backgrounds consisted of social services, chaplaincy, nursing, medicine, surgery, and case management. For the purpose of this paper, we focus on nurses' moral distress.

\subsection{Setting}

This study was conducted in a tertiary care center located in Houston, Texas. We interviewed healthcare professionals from two intensive care units, one with a medical focus and the other with a surgical focus. The interviews were conducted 
in a private meeting area. The interviews were semi-structured in nature, and a question guide was developed to frame the conversation.

\subsection{Analysis}

The interviews were audio-recorded, transcribed, double-coded, and analyzed using content thematic analysis. Data collection continued until new data no longer provided additional information, a condition referred to as saturation ${ }^{[18,19]}$. Analysis of the qualitative interviews was done in three stages: open coding, axial coding, and selective coding. The final coding scheme was developed by consensus and used for analysis. The interviews were double-coded and then reconciled to ensure accuracy.

\section{Results}

In this study, we interviewed thirteen nurses, who had a range of 3-30 years experience, with an average of 9 years of experience. There were 7 nurses from the medical unit and 6 nurses from the surgical unit. All nurses were female because no male nurses were identified as being heavily involved in the cases selected. This is a limitation we readily acknowledge. Below, we describe nurses' perceptions of their multifaceted professional roles as patient advocate, intermediary, and “preparer." We describe how unit or team dynamics can impede job responsibilities, and the impact these dynamics have on perceptions of moral distress.

\subsection{Patient advocate}

Nurses highlighted their role as patient advocate, "You're always taught from nursing school that you're an advocate for your patient, and that should be your main concern...if you feel like it's something that's best for your patient, stand up for your patient and do the proper thing, the appropriate thing." Several nurses felt a primary responsibility of being a patient advocate was to "listen closely" to patient or surrogates' questions about long-term clinical goals or clinical endpoints. Unlike many other healthcare professionals, intensive care unit nurses spend a significant amount of time with a particular patient, giving them the advantage of building a close connection with patients or families. Because of this close connection, nurses reported that patients and families tend to be more forthcoming with them than other healthcare professionals about when "enough is enough" as demonstrated by this nurse's report: "So when I pick up between the lines, that's when I really feel like I need to be bold and say what the patient wants to be said."

\subsection{I ntermediary}

Nurses also reported viewing themselves as an intermediary between the patient or family and the doctors. One nurse described her job as "having to be an in-between kind of person...[S]ometimes we as nurses are the human factor that bridges that gap between the patients and other team members...if I did any disservice to [the patient], it was keeping him on the side of being the patient, a receptor of treatments... and not allowing him to be a person.”

In their role as intermediary, many nurses reported that a key task for them was to "distill” the clinical messages and be a "synthesizer" of the diverse and conflicting messages from various medical consultants, "Sometimes I think...the role of the bedside nurse is to kind of pull it all together. And years ago, we had more control of that in perhaps smaller institutions, but now we're so specialized that we have all these other people.” This included stripping medical language and translating into lay language for patients or families: "You have to talk to the family and to the patient in a language they can understand. The [patients] cannot go scientific.”

\subsection{Preparing families}

When distilling or synthesizing different messages from the interviews, nurses reported that part of their job was to explain and prepare patients or families for different trajectories, including the possible outcome of death, "[When] the talking has been done, I would prepare them for [the course], because there are going to be lots of ups and downs. It's not going to be 
straightforward, and there may not be a good outcome no matter what we do." In preparing families, they can explore with patients or families' their perspective of what they consider to be a good death, "I have found...that if I'm brave enough to approach the subject [of dying]...it really opens the door for the patient to explore their feelings and how they-start working through how they want things to be. So it's up to my bravery factor and my willingness to go there."

One commonly-expressed sentiment was that nurses' abilities to communicate with patients or families are often constrained by team dynamics. As one nurse described it, "The doctors want to do this, and they have valid reasons for doing this, whereas the family wants to do this, and they have their valid reasons for doing this, and they are in discord. I agree with the family in their request, but I also agree with the doctors-in a way...It puts me in the middle." Exploring the patient's perspective can create a feeling of "being caught in the middle" especially when this perspective conflicts with the treatment goals of the medical team. This experience of divided loyalty disrupts the nurse's sense of professionalism and limits his/her ability to "prepare" patients and families for possible trajectories and clinical outcomes. Nurses explicitly recognize the importance of maintaining good relationships with the physicians, which may greatly impact their subsequent ability to communicate with patients or families:

"You're in a precarious position. How far do you go with the family when you've still got this other part [good relations with the healthcare team] you're responsible for...Then we have the family that is completely oblivious to what is going on, but [the nurses are] not, and sometimes the surgeons want to keep going and keep going, and we feel like our hands are tied.”

\subsection{Team dynamics}

A prominent theme in our interviews was nurses' perception of the importance of communication and the impact it has on quality of care. Nearly all nurse-interviewees attributed "prolonged deaths" to a lack of communication between consulting services and the inability to create a consensus on patients' clinical needs and long-term goals:

“Oncology says: ‘Oh, your mom is getting better.' Primary team says, 'No, she’s not getting better.' Infection team says, 'Oh, the infection is better.' So that is a problem, and it's always going to be a problem. Each [consultant] does one part of the system, and each one has different opinions...If you tell the family that one part of the body is working, they're going to hold onto that. One thing that's going right, they're going to hold onto that.

Several nurses volunteered that families would have greater trust in healthcare professionals if team members were unified and consistent in their message, allowing families to deescalate treatments or shift to comfort care measures (where appropriate). Nurses reported that, if greater efforts were taken to minimize what one nurse referred to as "communication fractures and breakdowns between team members," the family would have a framework to process consistent information which, in turn, might allow for a timely transition to comfort-only interventions.

Therefore, the primary source of moral distress for our interviewees was their explicit recognition that, when they identify with the humanity of specific patients, they are unable to adequately protect their professional boundaries. This can be especially acute when patients experienced a prolonged dying process. Their core sense of professional responsibilities to be a patient advocate, serve as an intermediary, or prepare patients and families was disrupted or impacted by a profound external influence: team dynamics. As a result, nurses felt that their professional and moral integrity was principally violated whenever they could not honor patient wishes or fully explore patient wishes because of team dynamics.

\section{Discussion}

There has been significant moral distress research describing the negative personal and institutional impact the distress causes in the workplace. Specifically, there has been a fourfold increase in MEDLINE citations on the topic in the last four years ${ }^{[2]}$. Despite this important research, there are several gaps in knowledge that must be addressed if we are to successfully intervene and mitigate healthcare professionals' moral distress. Of note, there has been a lack of investigation 
into the interconnections between individuals, unit/team, team-patient/ family relationships, self-awareness and system factors, and their relationship to the root causes of moral distress ${ }^{[3,6,17]}$.

We explored the interconnections between individual and team factors by interviewing clinicians in two different ICUs. We believe this is the first moral distress study to use case report methodology, allowing for a rich exploration of how the case unfolded and the points of moral distress. Our findings support many widely-held intuitions about nurses' moral distress and support previous research in many respects. For instance, previous research has illuminated that communication issues and beliefs about prolonged suffering are common root causes of moral distress ${ }^{[2,10,20,21]}$. Previous research has also suggested that nurses might be more susceptible to moral distress than other healthcare professionals because the profession is heavily dominated by females ${ }^{[15,21]}$. These nurses are often at the bedside, occasioning more frequent interactions with various parties. This direct patient exposure increases the personal intensity of the patient-nurse relationship ${ }^{[4,13]}$.

We move beyond previous research by highlighting how our study differs from previous work and how it adds to the cumulative understanding of moral distress in significant ways. The most striking aspect of our results is that is adds a previously-unexplored dimension: our nurse-interviewees unanimously believed that inconsistent messages between healthcare team members can frustrate nurses' abilities to work with patients or families concerning goals of care, leading to prolonged lengths of stay, escalations in treatment, and nursing moral distress.

A recent New England Journal of Medicine Perspective piece by Stavert and Lott discusses the "bystander effect" in medical care ${ }^{[22]}$. Stavert and Lott opine that the larger the group of medical consultants involved in a case, the more likely it is that each of them will defer the onus of decision making responsibilities to someone else. Using a clinical example, the authors suggest that frequent physician-to-physician handoffs, ambiguity over who is truly the "captain" of the case, and the frequent use of specialized consultants all contribute to decay in coordination of care, colloquially referred to as “medical purgatory" ${ }^{[22]}$. Our nurse-interviewees provide an empirical basis that support Stavert and Lott’s anecdotal report.

In addition, our nurse-interviewees reflect on the impact the "bystander effect" has on their sense of professional integrity, multidimensional collaborative role and residual moral distress. In vivid language, the nurses explicitly acknowledge the complex emotions they feel when they walk a thin line between acquiescing to a physician and respecting the patient's wishes, frequently using terms like "stuck in the middle," "feeling torn," "precarious position," or "tight-rope." Our nurse-interviewees discussed feeling this tension in the end-of-life context, where physicians believe continuation of aggressive therapies is warranted and patients or families indicate a preference for de-escalation of treatments. This conflict resulted in nurses' acute sense of moral failure and guilt in not honoring patient wishes or being constrained from fully exploring patient wishes.

From our interviews, we theorize that nursing staff may experience moral distress on a more personal level, not only because of the depth of their daily interactions with multiple parties, but perhaps they experience a sense of unfulfilled moral duty - a duty to respond to patient wishes. In this way, their empathy may be a component of distress in that they are a receptacle of the patient's/family's sense of angst. This interpretation is supported by the nurses' vivid descriptions of the unfolding of the cases. During the interviews, it appeared that the nurses actually relived the cases, often reflected in crying and enumerating specific details of interactions between healthcare professionals or between healthcare professionals and family members. Many nurses, for instance, actually used direct quotes from these interactions, saying they actually recalled the exact conversation. Reliving stories may be a component of the residual moral distress ${ }^{[2]}$. The passage of time did not seem to provide a sufficient perspective or mechanism to heal the individual anguish.

Another finding from our study is that our results departed from previous work on moral residue. A positive correlation between years of nursing practice and moral distress intensity scores was found in a study by Elpern, Covert, and Kleinpell ${ }^{[21]}$. While we did not directly test for moral residue (to our knowledge, there is no direct measure of moral 
residue), we question its generalizability. Our interview guide incorporated questions about moral residue, and we found no qualitative connection between years of nursing practice and moral residue. In fact, most nurses reported that they had higher moral distress intensities as younger professionals, which many attributed to a "lack of knowledge" or "familiarity" with clinical subtleties. If moral residue indeed exists, one would expect to find the inverse of what our interviewees reported.

The question then becomes: are there any practical strategies that might mitigate nurses' moral distress ${ }^{[3,17]}$ ? When asked this question, our interviewees said they find talking to other healthcare professionals helpful. Nearly all of them said they only find "venting" or "talking" helpful when they can talk to someone who is in healthcare and can relate to morally distressing situations. For this reason, we believe there is a potential role for bioethicists, social workers and chaplains to recognize symptoms of moral distress and allow nurses to openly talk through debriefing sessions ${ }^{[23]}$. The process of interviewing these nurses allowed a mechanism for reflection, awareness, re-enactment, and analysis, through supportive interview techniques ${ }^{[17]}$. A supportive and nonjudgmental interviewing approach might lay a foundation for a different response when a nurse is confronted with a future difficult situation. Part of an interviewing and debriefing session should be to provide nurses with validation that they are not committing a moral failure or error.

Another practical strategy nurses have found effective in ameliorating moral distress is effective mentoring networks ${ }^{[12,14]}$. Many nurses reported that they lean on charge nurses or more senior nurses to be mentors to them. However, nurses reported that the mentor cannot be judgmental and should not have supervisory authority over the mentee. Otherwise, the mentee nurse would feel reluctant to willingly disclose her feelings. Finally, supportive relationships between healthcare professionals in different disciplines can often mitigate distress. In one of the units, nearly all of the nurses said that "team members provide a united front," which enables them to feel a collective sense of shared moral distress and suffering. This gives meaning to the adage, “misery loves company.” Collective moral distress may provide a sense of moral resilience.

\section{Conclusion}

We sought to explore interconnections between individual and team factors involving moral distress. Our findings reflect a complicated relationship between nurses' perceptions of their role responsibilities, and the impact team dynamics has on nurses' abilities to perform these roles. A significant finding of our study was that nurses believe team dynamics frustrate their abilities to work with patients or families concerning goals of care. When they cannot fully explore patient wishes or cannot act upon patient wishes, often demonstrated by prolonged hospitalizations or protracted deaths, nurses feel they morally fail their patients.

Future research should explore whether "medical purgatory" is experienced in domains other than end-of-life, as well as the impact this has on quality of care and cost considerations. Although heightened recognition of this issue may help to minimize its impact, our research suggests that the emotional costs of medical purgatory may be profound. Nearly all of our interviewees revealed that they physically and emotionally "distance" themselves from patients and families as a way to accommodate moral distress. Conceivably, quality of care is greatly impacted if distancing occurs at the time patients need healthcare professionals the most.

\section{References}

[1] Jameton A. Nursing Practice: The Ethical Issues. Englewood Cliffs, NJ: Prentice Hall; 1984. PMid:10265936

[2] Epstein EG, Hamric AB. Moral distress, moral residue, and the crescendo effect. J Clin Ethics. 2009 ; 20 (4): 330-42. PMid:20120853

[3] Hamric AB. Empirical research on moral distress: issues, challenges, and opportunities. HEC Forum Interdiscip J Hosp Ethical Leg Issues. 2012 Mar; 24(1): 39-49. 
[4] Hamric AB. Moral distress and nurse-physician relationships. Virtual Mentor VM. 2010; 12(1): 6-11. PMid:23140776 http://dx.doi.org/10.1001/virtualmentor.2010.12.1.ccas1-1001

[5] Wilkinson JM. Moral distress in nursing practice: experience and effect. Nurs Forum (Auckl). 1988; 23(1): 16-29. http://dx.doi.org/10.1111/j.1744-6198.1987.tb00794.x

[6] Lützén K, Dahlqvist V, Eriksson S, Norberg A. Developing the concept of moral sensitivity in health care practice. Nurs Ethics. 2006 Mar; 13(2): 187-96. PMid:16526152 http://dx.doi.org/10.1191/0969733006ne837oa

[7] Corley MC, Elswick RK, Gorman M, Clor T. Development and evaluation of a moral distress scale. J Adv Nurs. 2001 Jan; 33(2): 250-6. PMid:11168709 http://dx.doi.org/10.1046/j.1365-2648.2001.01658.x

[8] Sporrong SK, Höglund AT, Hansson MG, Westerholm P, Arnetz B. "We are white coats whirling round"--moral distress in Swedish pharmacies. Pharm World Sci PWS. 2005 Jun; 27(3): 223-9. PMid:16096892 http://dx.doi.org/10.1007/s11096-004-3703-0

[9] Sporrong SK, Höglund AT, Arnetz B. Measuring moral distress in pharmacy and clinical practice. Nurs Ethics. 2006 Jul; 13(4): 416-27. PMid:16838572 http://dx.doi.org/10.1191/0969733006ne880oa

[10] Hamric AB, Blackhall LJ. Nurse-physician perspectives on the care of dying patients in intensive care units: collaboration, moral distress, and ethical climate. Crit Care Med. 2007 Feb; 35(2): 422-9. PMid:17205001 http://dx.doi.org/10.1097/01.CCM.0000254722.50608.2D

[11] Elpern EH, Silver MR. Improving outcomes: focus on workplace issues. Curr Opin Crit Care. 2006 Oct; 12(5): 395-8. PMid:16943715 http://dx.doi.org/10.1097/01.ccx.0000244116.31624.b3

[12] Rushton CH. Defining and addressing moral distress: tools for critical care nursing leaders. AACN Adv Crit Care. 2006 Jun; 17(2): 161-8. PMid:16767017 http://dx.doi.org/10.1097/00044067-200604000-00011

[13] Corley MC. Nurse moral distress: a proposed theory and research agenda. Nurs Ethics. 2002 Nov; 9(6): 636-50. PMid:12450000 http://dx.doi.org/10.1191/0969733002ne557oa

[14] Corley MC, Minick P. Moral distress or moral comfort. Bioeth Forum. 2002; 18(1-2): 7-14.

[15] Repenshek M. Moral distress: inability to act or discomfort with moral subjectivity? Nurs Ethics. 2009 Nov; 16(6): 734-42. PMid:19889914 http://dx.doi.org/10.1177/0969733009342138

[16] Corley MC. Moral distress of critical care nurses. Am J Crit Care Off Publ Am Assoc Crit-Care Nurses. 1995 Jul; 4(4):280-5.

[17] Beumer CM. Innovative solutions: the effect of a workshop on reducing the experience of moral distress in an intensive care unit setting. Dimens Crit Care Nurs DCCN. 2008 Dec; 27(6): 263-7. PMid:18953194 http://dx.doi.org/10.1097/01.DCC.0000338871.77658.03

[18] Martin DK, Pater JL, Singer PA. Priority-setting decisions for new cancer drugs: a qualitative case study. Lancet. 2001 Nov 17; 358(9294): 1676-81. http://dx.doi.org/10.1016/S0140-6736(01)06714-9

[19] Singer PA, Martin DK, Giacomini M, Purdy L. Priority setting for new technologies in medicine: qualitative case study. BMJ. 2000 Nov 25; 321(7272): 1316-8. PMid:11090513 http://dx.doi.org/10.1136/bmj.321.7272.1316

[20] Epstein EG. End-of-life experiences of nurses and physicians in the newborn intensive care unit. J Perinatol Off J Calif Perinat Assoc. 2008 Nov; 28(11): 771-8. PMid:18596708 http://dx.doi.org/10.1038/jp.2008.96

[21] Elpern EH, Covert B, Kleinpell R. Moral distress of staff nurses in a medical intensive care unit. Am J Crit Care Off Publ Am Assoc Crit-Care Nurses. 2005 Nov; 14(6): 523-30.

[22] Stavert RR, Lott JP. The bystander effect in medical care. N Engl J Med. 2013 Jan 3; 368(1): 8-9. PMid:23281972 http://dx.doi.org/10.1056/NEJMp1210501

[23] Gordon EJ, Hamric AB. The courage to stand up: the cultural politics of nurses' access to ethics consultation. J Clin Ethics. 2006; 17(3): 231-54. PMid:17186936 